\title{
Visualization of schistosomiasis snail habitats using light unmanned aerial vehicles
}

\author{
Andrew J. Chamberlin, ${ }^{1}$ Isabel J. Jones, ${ }^{1}$ Andrea J. Lund, ${ }^{2}$ Nicolas Jouanard,,${ }^{3,4}$ Gilles Riveau, ${ }^{3}$ \\ Raphaël Ndione, ${ }^{3}$ Susanne H. Sokolow, ${ }^{1}$ Chelsea L. Wood, ${ }^{5}$ Kevin D. Lafferty, ${ }^{6,7}$ \\ Giulio A. De Leo ${ }^{1}$ \\ ${ }^{1}$ Hopkins Marine Station, Stanford University, Pacific Grove, CA, USA $;{ }^{2}$ Emmett Interdisciplinary Program in \\ Environment and Resources, Stanford University, Stanford, CA, USA; ${ }^{3}$ Biomedical Research Center EPLS, \\ Saint Louis, Senegal; ${ }^{4}$ Station d'Innovation Aquacole, Saint Louis, Senegal; ${ }^{5}$ School of Aquatic and Fishery \\ Sciences, University of Washington, Seattle, WA; ${ }^{6}$ Western Ecological Research Center, United States \\ Geological Survey, CA, USA; ' Marine Science Institute, University of California, Santa Barbara, CA, USA
}

\begin{abstract}
Schistosomiasis, or "snail fever", is a parasitic disease affecting over 200 million people worldwide. People become infected when exposed to water containing particular species of freshwater
\end{abstract}

Correspondence: Andrew J. Chamberlin, Hopkins Marine Station, Stanford University, Pacific Grove, CA, USA.

E-mail: andy.chamberlin@gmail.com

Key words: Drones; health assessment; risk-mapping; schistosomiasis; Senegal; spatio-temporal visualization.

Video link: https://drive.google.com/file/d/1HreioJXI9H-DrL_lekIEre $\mathrm{P} 4 \mathrm{e} 9 \mathrm{r}-\mathrm{F} 2 \mathrm{ha} /$ view? usp=sharing

Acknowledgments: The authors were supported by a grant from the Bill and Melinda Gates Foundation (OPP1114050), a grant from the National Institutes of Health (R01TW010286), and a grant from the National Science Foundation (1414102). AJC, IJ, SHS, and GADL were partially supported by a 2018 Environmental Venture Program grant from the Stanford University Woods Institute for the Environment and a GDP SEED grant from the Freeman Spogli Institute at Stanford University. AJL was supported by the Davis Family E-IPER Fellowship and the James and Nance Kelso Fellowship through the Stanford Interdisciplinary Graduate Fellowship (SIGF) program at Stanford University. CLW was supported by the Michigan Society of Fellows at the University of Michigan, a University of Washington Innovation Award from the UW Innovation Imperative, and a Sloan Research Fellowship from the Alfred P. Sloan Foundation. Any use of trade, product, or firm names in this publication is for descriptive purposes only and does not imply endorsement by the US Government. GADL has been partially supported also by NSF grants ICER-2024383 and DEB2011179.

Conflict of interests: The authors declare no potential conflict of interests.

Received for publication: 28 September 2019.

Accepted for publication: 13 February 2020.

(C) Copyright: the Author(s), 2020

Licensee PAGEPress, Italy

Geospatial Health 2020; 15:818

doi:10.4081/gh.2020.818

This article is distributed under the terms of the Creative Commons Attribution Noncommercial License (CC BY-NC 4.0) which permits any noncommercial use, distribution, and reproduction in any medium, provided the original author(s) and source are credited. snails. Habitats for such snails can be mapped using lightweight, inexpensive and field-deployable consumer-grade Unmanned Aerial Vehicles (UAVs), also known as drones. Drones can obtain imagery in remote areas with poor satellite imagery. An unexpected outcome of using drones is public engagement. Whereas sampling snails exposes field technicians to infection risk and might disturb locals who are also using the water site, drones are novel and fun to watch, attracting crowds that can be educated about the infection risk.

\section{Background}

Searching for snails in parasite-contaminated water is dangerous and labour-intensive; yet it has long been the most effective way to assess the distribution of infection risk for human schistosomiasis, a trematode parasite that uses specific freshwater snails as obligate intermediate hosts (Le et al., 2015). Schistosomiasis affects more than 200 million people with 800 million at risk (WHO, 2016), and is responsible for the loss of more than 1.43 million Disability-Adjusted Life Years (DALYs) annually (GBD 2017 DALYs and HALE Collaborators, 2018; WHO, 2016). Infection can damage the liver or the urogenital organs depending on the species of the parasite, and it can also cause anaemia, stunted growth, cognitive impairment and sometimes bladder cancer (King et al., 2005), with children and young adults bearing a disproportionate disease burden (Barry et al., 2013). Other trematodes transmitted through aquatic snails can also infect humans, including food-borne trematodes, and still others, like non-human schistosomes and Fasciola, can cause considerable damage to livestock, often the most valuable asset in subsistence economies (WHO, 2018).

Schistosomiasis control programmes often seek to interrupt schistosome transmission cycles by targeting adult stages of the parasite through treating infected people with Mass Drug Administration (MDA) programmes (Sokolow et al., 2016); by targeting larval stages of the parasite through snail control using molluscicides, biological control (Sokolow et al., 2015); or habitat modification through environmental management (Boelee and Laamrani, 2004); or through integrated approaches. A historical analysis of global control efforts reveals that snail control interventions were essential to many successful control campaigns (Sokolow et al., 2016) and will be most efficient when it targets areas and seasons where snails are common.

Because snails often live on aquatic plants (Cogels et al., 
1997; Wood et al., 2019), high-definition aerial imagery taken with a drone can be used to precisely measure the extent of suitable habitats for snail hosts and infer snail abundance across large areas, including areas that are unsafe or difficult to access by technicians, and deep waters that are generally excluded from sampling in field-based snail surveys due to safety concerns. Drone-acquired videos are also a powerful tool to engage local stakeholders and public health officers, to raise awareness of the risks of using potentially contaminated water and to spur measures aimed at controlling disease transmission.

We explored using drones to define snail habitats in an area that is hyper-endemic for schistosomiasis - the lower basin of the Senegal River. The longitudinal study assessed annual re-infection rates of $\sim 1,500$ school-age children from 16 villages located on the shores of the Senegal River and two of its tributaries, the Lampsar and N'Galam Rivers, as well as a natural overflow lake, the Lac de Guiers, all of which are near Senegal's northern border with Mauritania (Sokolow et al., 2015). Preliminary analysis of the epidemiological data gathered in 2016-2017 shows that schistosomiasis prevalence ranged between 42 and $99 \%$ for the Schistosoma haematobium species that causes urogenital schistosomiasis and between 0 and $80 \%$ for $S$. mansoni which causes intestinal schistosomiasis among enrolled schoolchildren (Sokolow et al., 2015).

We evaluated associations between snails and various freshwater habitats (including open water, floating and submerged vegetation and emergent vegetation) at the water-access points of 16 villages. After baseline infection prevalence in enrolled schoolchildren was measured, field surveys were conducted three times a year for two subsequent years beginning in May 2016, to track the inter-annual and seasonal fluctuations in snails and their habitats in this region (Sturrock et al., 2001). Knowing the specific plant species preferred by snails (Wood et al., 2019) allowed us to estimate snail presence, density and abundance from drone imagery.

\section{Field data}

\section{Drone surveys}

Starting in January 2017, we matched malacology surveys with aerial photos and videos taken with a low cost $(<$ USD1500 in 2017) commercial DJI Phantom 4 drone (DJI, 2017) factoryequipped with a 12.4-megapixel camera with $\mathrm{f} / 2.8$ and $1 / 2.3$ " CMOS sensor. It took $30 \mathrm{~min}$ for a drone operator and one field technician to gather imagery and video at a site. Several droneassisted photos were taken from the zenith position of each water access point at 20-500 $\mathrm{m}$ above ground level. Ground reference points, in our case consisting of square pieces of black fabric with red tape and an orange cone at the centre, with associated Global Positioning System (GPS) points, were identified at each water access point and later used to produce orthophotos by using the open source software OpenDroneMap version 0.2 (OSM, 2019). Spatial coordinates of reference points were recorded using a highaccuracy GPS Trimble R1 RTK GNSS (Trimble, 2019). Estimated pixel resolution of drone-assisted photos ranged between 0.5 $\mathrm{cm} /$ pixel at $20 \mathrm{~m}$ above ground level and $20 \mathrm{~cm} /$ pixel at $500 \mathrm{~m}$ above ground level.

By using colour thresholding, pixel analysis and the expertise of field technicians extensively trained in the identification of vegetation in the study region, it was possible to categorize different vegetation genera from the high-resolution aerial images using the image processing software ImageJ (ImageJ, 2019). During the project, the team developed technical expertise in remote-sensing image classification and switched to using object-based image analysis and supervised machine-learning using the software eCognition (Trimble Inc., Sunnyvale, CA, USA). ArcGIS (ESRI, Redlands, CA, USA) was used for correcting small errors in georeferenced drone imagery, computing surface areas of vegetation types and quantifying change over time at each study site.

\section{Results}

Although reeds often obscured the view from the ground, the analysed drone photos revealed potential snail habitats in both shallow and deep waters surrounding a muddy-bottomed area at water-access points used for collecting water, washing animals, bathing and doing laundry. This vastly extends the information that can be gathered by technicians surveying transmission sites on the ground. Drone imagery can be used to quantify the area of suitable snail habitats in transmission sites, which has been shown to be a superior predictor of human disease risk over fine-scale data on snail abundance or density collected by hand within the same area (Wood et al., 2019).

During our snail surveys, we documented floating vegetation infested with schistosome-infected snails, once as far as $100 \mathrm{~m}$ from the shore, in $>2 \mathrm{~m}$ of water, suggesting that snails tend to favour vegetated areas and that these areas might extend beyond what is easily sampled by technicians. Guided by drone-acquired aerial images, we speculate that offshore patches of suitable snail habitat might contribute to the observed high infection levels in school-age children in nearby villages, even where intermediate host snails were not found in the near-shore habitats. We plan to investigate this hypothesis by sampling snails further from shore and in deeper waters.

Drone images documented dramatic seasonal changes in the shoreline vegetation at some sites, while the vegetation was static at others. After villagers, clear vegetation to ease access, open water habitat (poor habitat for schistosome-bearing snails in this region) is first re-colonized by Ceratophyllum spp. (good habitat for snails), which can then become substrate for Ludwigia spp. (intermediate-quality snail habitat), which are then succeeded by the dominant cattail reed Typha spp. (poor- to intermediate-quality snail habitat). Data are currently being analysed across the entire scale of our study region to verify this pattern. Associations between snail survey data and human parasitological data over time will be used to develop a seasonal transmission index at a fine spatial and temporal scale.

The strong association between snails and floating vegetation suggests that clearing vegetation could be an effective intervention for schistosomiasis control. Drone-generated vegetation maps were used to design vegetation removal experiments in a selection of the study sites and identify the projected labour costs for removing vegetation at a site.

Technicians sampling snails are exposed to risk of water contact and can disturb local people using the water site for daily chores or disturb domestic and wild animals. On the contrary, the drone team was greeted with great curiosity and enthusiasm by local village residents, scientific staff and the malacology and human parasitology team, who liked watching drones fly and enjoyed aerial views of their villages. Training local graduate stu- 

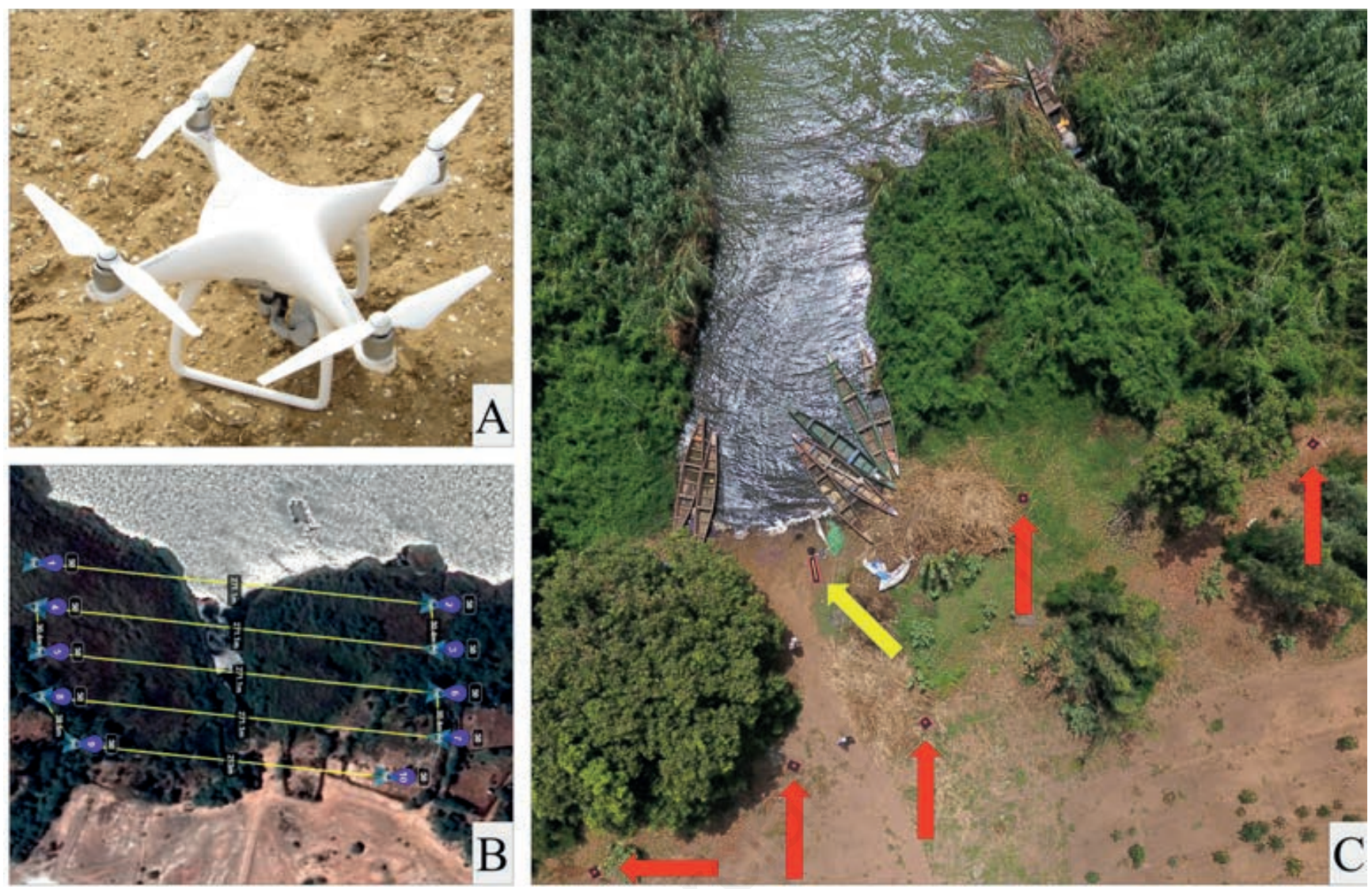

Figure 1. Use of drones for aerial mapping surveys. A) DJI Phantom 4 drone; B) drone flight plan for image gathering; C) overhead image of a site with clearly marked ground reference points (red arrows) and a meter stick for scaling (yellow arrow).

dents and technicians to fly drones improved capacity and helped transfer a new technology to Senegal. Finally, the aerial photos and videos led to conversations with rural villagers, Senegalese public health officers, students, researchers and the general public - and thereby increased awareness about risk of infection associated with particular aquatic landscapes in Senegal.

\section{Outlook}

We present a drone-based approach for visualizing areas at risk for schistosomiasis transmission in remote settings where physical access is limited or impossible. In addition, drone imagery and videos can increase awareness among local villagers, public health authorities and policy makers. Expanding the light-weight, inexpensive and uncomplicated UAV technology will not only help to increase understanding of the environmental drivers of diseases but also produce measurable benefits toward scaling up the results of on-the-ground malacology surveys, tracking seasonal changes in disease risk and informing potential control efforts in a highly dynamic environment.

\section{References}

Barry MA, Simon GG, Mistry N, Hotez PJ, 2013. Global trends in neglected tropical disease control and elimination: Impact on child health. Arch Dis Child 98:635-41. doi:10.1136/archdischild-2012-302338

Boelee E, Laamrani H, 2004. Environmental control of schistosomiasis through community participation in a Moroccan oasis. Trop Med Int Health 9:997-1004. doi:10.1111/j.13653156.2004.01301.x

Cogels FX, Coly A, Niang A, 1997. Impact of dam construction on the hydrological regime and quality of a Sahelian lake in the River Senegal basin. Regulated Rivers: Res Manag 13:27-41. doi:10.1002/(SICI)1099-1646(199701)13:1<27::AIDRRR421>3.0.CO;2-G

DJI, 2017. Phantom 4, https://www.dji.com/phantom . Accessed 30th Apr 2019

GBD 2017 DALYs and HALE Collaborators, 2018. Global, regional, and national disability-adjusted life-years (DALYs) for 359 diseases and injuries and healthy life expectancy (HALE) for 195 countries and territories, 1990-2017: a systematic analysis for the Global Burden of Disease Study 2017. Lancet 392:1859-1922. doi:10.1016/S0140-6736(18)32335-3 ImageJ, 2019. https://imagej.nih.gov/ij. Accessed 30th Apr 2019 King CH, Dickman K, Tisch DJ, 2005. Reassessment of the cost of 
chronic helmintic infection: A meta-analysis of disability-related outcomes in endemic schistosomiasis. Lancet 365:1561-9. doi:10.1016/S0140-6736(05)66457-4

Le T-L, Sokolow SH, Hammam O, Fu C-L, Hsieh M, 2015. Pathogenesis of Human Schistosomiasis. Human Emerging and Re-emerging Infections: Viral Parasitic Infect 1:481-504. doi:10.1002/9781118644843.ch25

OSM, 2019. OpenStreetMap contributors. https:/www.openstreetmap.org, Accessed 30th Apr 2019

Sokolow SH, Wood CL, Jones IJ, Swartz SJ, Lopez M, et al., 2016. Global Assessment of Schistosomiasis Control Over the Past Century Shows Targeting the Snail Intermediate Host Works Best. PLoS Negl Trop Dis 10:e0004794. doi:10.1371/ journal.pntd.0004794

Sokolow SH, Huttinger E, Jouanard N, Hsieh MH, Lafferty KD, et al., 2015. Reduced transmission of human schistosomiasis after restoration of a native river prawn that preys on the snail intermediate host. Proc Nat Acad Sci U S A 112:9650-5. doi:10.1073/pnas.1502651112

Sturrock RF, Diaw OT, Talla I, Niang M, Piau JP, Capron A, 2001.
Seasonality in the transmission of schistosomiasis and in populations of its snail intermediate hosts in and around a sugar irrigation scheme at Richard Toll, Senegal. Parasitol 123:S7789. doi:10.1017/ s0031182001008125

Trimble, 2019. GPS Trimble R1 RTK GNSS. https://geospatial. trimble.com/products-and-solutions/trimble-r1 . Accessed 30th Apr 2019

Wood CL, Sokolow S, Jones I, Chamberlin AJ, Lafferty KD, et al., 2019. Precision mapping of snail habitat provides a powerful indicator of human schistosomiasis transmission. Proc Nat Acad Sci U S A 116:23182-91. doi: 10.1073/pnas.1903698116

WHO, 2016. Schistosomiasis: Number of people treated worldwide in 2014. Weekly Epidemiol Rec 91:53-60. Available from: https://www.who.int/schistosomiasis/resources/who_wer 9105/en/

WHO, 2018.Human fascioliasis: review provides fresh perspectives on infection and control. Available from: https://www. who.int/neglected_diseases/news/fascioliasis-review-provides-new-perspectives-infection-control/en/ 